\title{
Finite Element Analysis Process Reuse Method based on Integrated Information Model
}

\author{
Dai Ye*, Lai Yi-Nan, Ren Sheng-Le and Ying Yu-Xiang \\ School of Mechanical \& Power Engineering, Harbin University of Science and \\ Technology, Heilongjiang Harbin 150080, China \\ *daiye312@163.com
}

\begin{abstract}
Aiming at the problems such as low efficiency of the simulation analysis, difficulty in information reuse between similar structures, an Finite Element Analysis (FEA) process reuse method based on the modular thought is put forward. The reuse process of FEA is described by the methods of module division and reorganization, and the FEA structural information is expressed using Extensive Makeup Language (XML). Macros and macro library technology of ANSYS are used to construct reusable modules. For the achievement of the module reuse in FEA process, the secondary development technology is conducted by combined with $V C++$ language with APDL parametric language. The approach is plied in nuclear power valve products through building FEA process reuse platform, which increased the efficiency of simulation analysis in valve products development.
\end{abstract}

Keywords: finite element; integrated information model; process reuse; valve product

\section{Introduction}

The finite element analysis (FEA) plays an important role in the field of engineering technology. In recent years, a number of experts and scholars at home and abroad give high attention on how to improve the quality and efficiency of FEA [1-4]. Finite element modeling contains many steps, including definition of analysis type, entity modeling, definition of unit type, definition of material properties, meshing, definition of constraint and load, and other links. The process of FEA is time-consuming and large amounts of data and information are difficult to reuse [5-9]. So it is important to study the process reuse method of FEA especially in series products development. Therefore, this paper focuses on of the process reuse method of FEA, and the modular thought is introduced into the finite element analysis process reuse, in order to solve the deficiency existing in the reuse technique of FEA.

\section{Reuse Knowledge Integration Model of FEA Process}

Figure 1 illustrates the reuse process of a traditional finite element analysis, it only achieve the inside reuse of the analysis process. This reuse way improves the efficiency of the finite element analysis on a certain extent, but it could not be able to implement the reuse analysis process for the similar topology models. In order to solve the shortage, it puts forward using $\mathrm{XML}$ language to describe the analysis knowledge among similar topology structures so as to realize the knowledge reuse of finite element analysis between similar models. 


\subsection{Definition of Finite Element Analysis Integration Model (FEAIM)}

In the field of knowledge reuse in the finite element simulation analysis, the process can be divided into two main parts: finite element operating knowledge reuse (FEA Operation), and the finite element parameters knowledge reuse (FEA Parameter). In order to fit the demand of product modularization in process reuse, finite element analysis integration model (FEAIM) is constructed by a representation of modularization.

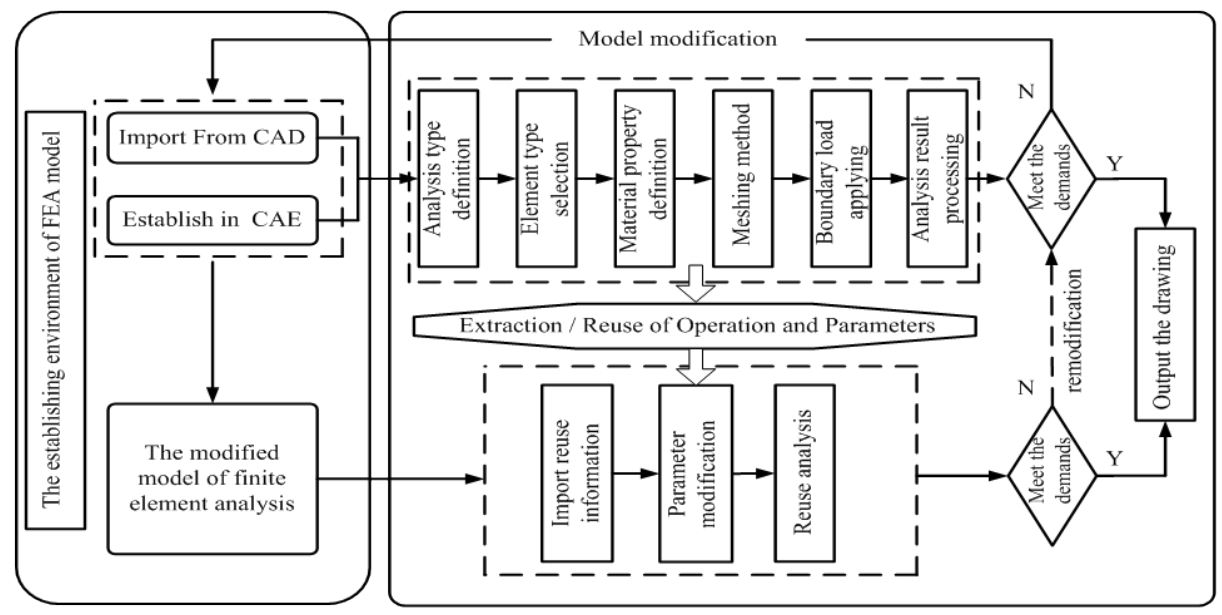

Figure 1. Reuse Process of Finite-element Analysis

Definition 1: If FEAIM be represented by $S$, then

$$
S_{\text {FEAIM }}=<F_{1}, F_{2}, F_{3}, \ldots \ldots, F_{n}>^{T}
$$

$S_{\text {FEAIM }}$ can be called the total model of FEAIM. $F_{i}(i=1,2, \ldots, n)$ is the subset of $S_{\text {FEAIM }}$, which contains two parts, namely $F_{i}=<O_{i}, P_{i}>, O_{i}$ represents a subset of information related to the specific operation, and $P_{\bar{i}}$ represents a subset of information related to the input parameters.

Definition 2: For the operation model $\boldsymbol{O}$, it can be defined as

$$
O=<O_{B}, O_{E}, O_{D}, O_{M}, O_{T}, O_{R}, O_{L}, O_{S}, \ldots \ldots>^{T}
$$

Where $O_{B}$ is solid modeling operations, $O_{E}$ is element selection operation, $O_{D}$ is Material property operation, $O_{M}$ is grid meshing operation, $O_{T}$ is analysis type operation, $O_{R}$ is boundary conditions operation, $O_{L}$ is load applying operation, and $O_{S}$ is processing operations of analysis results.

Definition 3: For the parameter model $P$, it can be defined as

$$
P=<P_{B}, P_{E}, P_{D}, P_{M}, P_{T}, P_{R}, P_{L}, P_{S}, \ldots \ldots>^{T}
$$

Where $P_{B}$ is solid modeling parameters, $P_{E}$ is element selection parameters, $P_{D}$ is Material property parameters, $P_{M}$ is grid meshing parameters, $P_{T}$ is analysis type parameters, $P_{R}$ is boundary conditions parameters, $P_{L}$ is load applying parameters, and $P_{S}$ is processing parameters of analysis results. 


\subsection{Model Information Expression based on XML}

This paper puts forward a method that solid model information is recorded using XML language, which has advantage of good data storage format and facilitating extension [10]. Model information expression by XML is more easier way to transfer the key parameters between CAD and CAE. With a certain gate valve body as an example, a part of valve body model information of XML description as below:

$<$ Model_Info name=" valve body " department=" engineering department " author ="David" date="2013-2-7">

$<$ Content Name=" Characteristics of the valve body " >

$<$ Attribute1 $>$

Body model features

$</$ Attribute1 $>$

$<$ Attribute2 $>$

Finite element analysis of valve body features

$</$ Attribute2 $>$

$</$ Content $>$

$<$ MI_valvebody $>$

$<$ Feature_info $>$

$<$ Attri_Valve name=" inside nominal diameter" weight $=$ "0.40" $>200</$ Attri_Valve $>$

$<$ Attri_Valve name $=$ " work stress" weight $=$ "0.30" $>30</$ Attri_Valve $>$

$<$ Attri_Valve name=" operating temperature" weight="0.30" > $600<$ /Attri_Valve $>$

$<$ Attri_Valve name $=" P_{D}$ " > carbon steel </Attribute $>$

$<$ Attri_Valve name=" connection type " > pipeline welding $</$ Attri_Valve $>$

$</$ Feature_info $>$

$<$ FEA_info $>$

$<$ Attri_Valve name $=$ " $O_{T}$ " $>$ Thermal coupling analysis $</$ Attri_Valve $>$

$<$ Attri_Valve name $=$ " solver " $>$ Ansys $<$ / Attri_Valve $>$

$</$ FEA_info $>$

$</$ MI_valvebody $>$

$</$ Model_Info $>$

\section{Reuse Process of Finite Element Numerical Simulation}

\subsection{The Generation of FEA Reuse Mode}

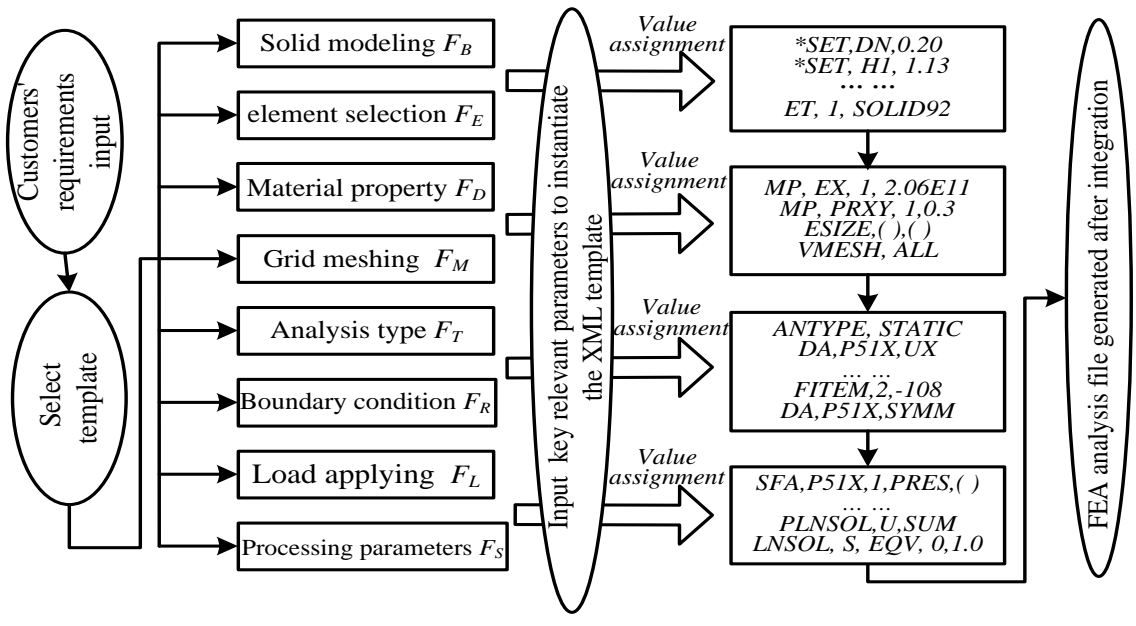

Figure 2. Data Transmission Flow Diagram of Reuse Process 
The process of generating the FEA reuse model, making the command stream processing of the typical parts standardization, contains numerical parameterization (such as characteristic parameter values, grid size, etc.), finite element operation parameterization (such as definition of the material properties, result reduction, etc.). In the XML Reuse template file of product typical parts, the three basic processes of pre-treatment, solving and post-processing need to be defined; and then XML drive the analysis template to transmit design model from CAD to CAE. It eventually would form the product reuse model of finite element analysis. In ANSYS environment, for instance, the generation of FEA reuse model is to compile the command files of FEA using ANSYS Parametric Design Language (APDL); and then according to each parameter name in the XML template file, the new parameter values can be wrote to APDL command file through the parameter name matches, the whole process of data transfer flow as shown in Figure 1.

\subsection{The Rapid Reuse Process of Finite Element Analysis}

The key point of reuse process as shown in Figure 3: one is to form a unified reuse data model, namely the reusable template under the XML definition; another one is the parameterized finite element modeling techniques. On the basis of the above key technologies, the reuse process can be generally described as:

Step 1: According to the design parameters provided by customers, the feature decomposition on the new products structure can be done, and then information expression using XML language for model could be recorded as knowledge file;

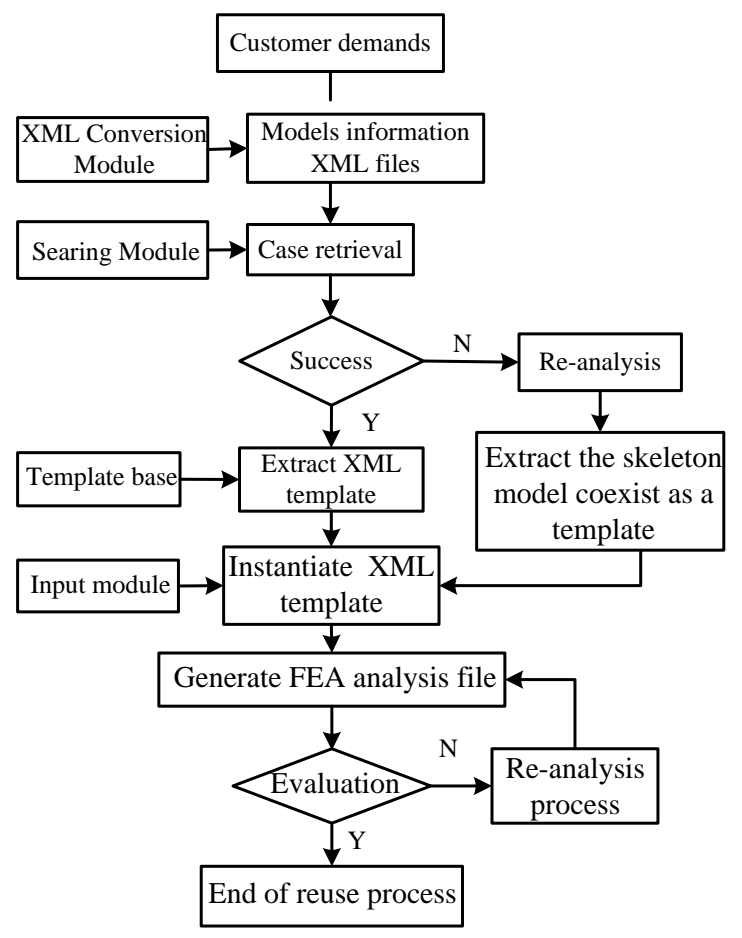

Figure 3. Rapid Analyses Reuse Process of FEA

Step 2: Using the new generated XML documents, according to the key information and weight, similarity calculation in case database can be carried out with the setting matching rules. Find out the highest similarity instance as a reusable template. If it do not 
meet the design requirements, modifying appropriately the matching rules to search more examples, until meet the design requirements; If there is no reuse instance, operators could make new finite element analysis for this structure and save as new instance;

Step 3: Modify the specific parameters of the instance model, and then pass the modified parameter value to the reuse template defined by XML so as to instantiate the template;

Step 4: Parse the instantiated XML file, and search the module element needed by FEA for the element (for different environment of FEA, the extracted module contents are different); Find out all of corresponding order contents, including $F_{T}$ (Analysis type), $F_{B}$ (Solid modeling), $F_{E}$ (Element selection), and so on; and then the order flow for FEA finally can be obtained, and finite element discrete model could be generated to carry out the solution, finally feedback the reports;

Step 5: For those analysis tasks does that do not meet the design requirements need to make re-design and re-analysis. In the reuse process, it also needs to apply the finite element method to solve in order to rapidly and accurately complete a large number of finite element analysis.

\section{Application of Reuse Analysis in Nuclear Power Valve}

Through the established reuse model of FEA finite element above, the whole FEA process of nuclear power valve products, including solid model, element type, material property, and so on, could be integrated in order to achieve the reuse process. This paper, taking a certain supercritical electric gate valve body for example, illustrates the whole reuse process of FEA. Assume that the customer demand content is: work pressure of the valve body is $27 \mathrm{MPa}$, work temperature is $550^{\circ} \mathrm{C}$, and nominal diameter is $D N=200 \mathrm{~mm}$, material is carbon steel, connection type to the piping system is weld. Structural mechanics analysis by using ANSYS needs to be done so as to determine if it meets safety standards

\subsection{Information Expression of Valve Body Model}

In accordance with the reuse steps as shown in Section 3.2 above, information expression first of the valve body model using XML language should be listed, its knowledge file is as follow:

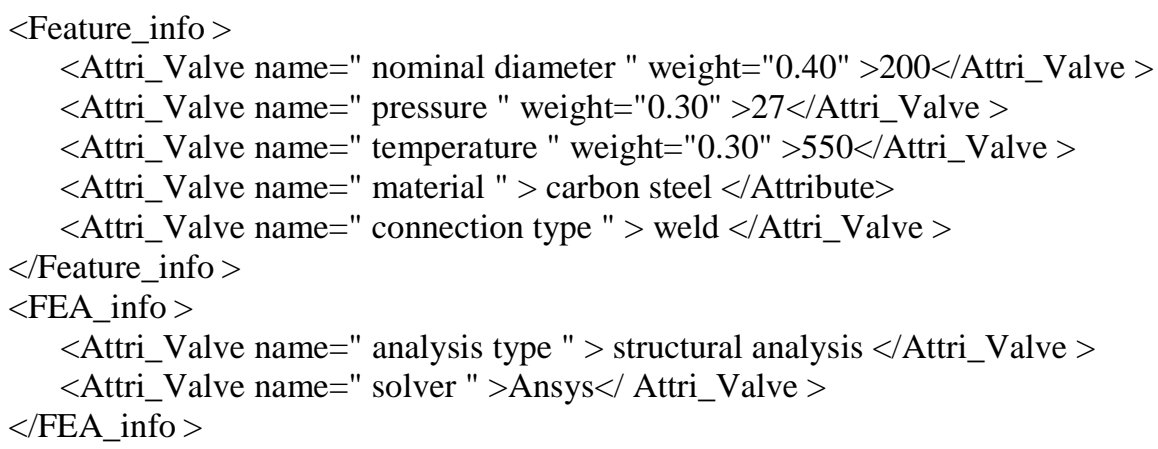

\subsection{Selection Process of Reuse Template}

In this paper, fuzzy similarity priority ratio can be used to describe the fuzzy similar order between target instance and each instance, the algorithm has detailed discussion in literature [11], and their details are not given here. Assume that there are 8 FEA reuse templates in template base, the attribute values of each template are as shown in Table $1, C_{0}$ is design 
object module. According to the qualitative attributes matching, template set $\left\{C_{1}, C_{3}, C_{6}\right.$, $\mathrm{C}_{7}$ \} meet the design requirements. According to the quantitative attributes there is no exact match template, so similarity calculation should be carried out. With nominal diameter as an example, the similarity sorting process is introduced as follow.

(1) Semantic Distance Calculation. According to the data in Table 1, it can be obtained:

$$
S D\left(a_{11}, a_{01}\right)=S D\left((D N)_{1},(D N)_{0}\right)=|175-200|=25
$$

Table 1. Template Property Parameter Table

\begin{tabular}{cccccc}
\hline Template & $\begin{array}{c}\text { DN } \\
/ \mathrm{mm}\end{array}$ & $\begin{array}{c}\text { Pressure } \\
/ \mathrm{MPa}\end{array}$ & $\begin{array}{c}\text { Temperature } \\
/{ }^{\circ} \mathrm{C}\end{array}$ & Material & $\begin{array}{c}\text { Connection } \\
\text { type }\end{array}$ \\
\hline$C_{0}$ & 200 & 27 & 550 & WCB & weld \\
$C_{1}$ & 175 & 10 & 350 & WCB & weld \\
$C_{2}$ & 150 & 40 & 400 & Cr12Mo1V1 & flange \\
$C_{3}$ & 275 & 15 & 500 & WCB & weld \\
$C_{4}$ & 200 & 30 & 600 & Cr12Mo1V1 & weld \\
$C_{5}$ & 150 & 25 & 250 & Cr12Mo1V1 & flange \\
$C_{6}$ & 250 & 40 & 650 & WCB & weld \\
$C_{7}$ & 300 & 20 & 200 & WCB & weld \\
$C_{8}$ & 350 & 35 & 550 & Cr12Mo1V1 & flange \\
\hline
\end{tabular}

(2) Fuzzy Similarity Priority Ratio. According to the algorithm of fuzzy similarity priority ratio, it can be obtained:

$$
S D_{13}^{1}=\frac{S D\left(a_{31}, a_{01}\right)}{\left[S D\left(a_{11}, a_{01}\right)+S D\left(a_{31}, a_{01}\right)\right]}=\frac{25}{[25+25]}=0.5
$$

Similarly, $S D_{p q}^{1}(p, q=1,3,6,7)$, therefore the matrix of fuzzy similar priority ratio correspond to the $D N$ can be obtained:

$$
S D(1)=\left[\begin{array}{cccc}
0 & 0.75 & 0.67 & 0.80 \\
0.25 & 0 & 0.4 & 0.57 \\
0.33 & 0.60 & 0 & 0.67 \\
0.20 & 0.43 & 0.33 & 0
\end{array}\right] \text {, and } S D(1)_{0.5}=\left[\begin{array}{cccc}
0 & 1 & 1 & 1 \\
0 & 0 & 0 & 1 \\
0 & 1 & 0 & 1 \\
0 & 0 & 0 & 0
\end{array}\right]
$$

That is when $\lambda=0.5, S D(1)$ could rule out the first column and the first line, and then the third order matrix can be obtained:

$$
S D(1)^{\prime}=\left[\begin{array}{ccc}
0 & 0.4 & 0.57 \\
0.6 & 0 & 0.67 \\
0.43 & 0.33 & 0
\end{array}\right]
$$

Repeating the process using each sectional set of $S D(1)^{\prime}$, it can be obtained that $T_{1}=\{1,3,2,4\}$. 
(3) Comprehensive Sorting of each Attribute. According to the sorting process as shown in Table 2, it can be obtained that $T_{2}=\{4,2,3,1\}, T_{3}=\{3,1,2,4\}$. And the Sequence similarity degree $t_{i}$ can be obtained:

$$
t_{1}=\sum_{j=1}^{3} \varpi_{j} t_{1 j}=0.4 \times 1+0.3 \times 4+0.3 \times 3=2.5
$$

Similarly, $t_{3}=2.1, t_{6}=2.3, t_{7}=3.1$. According to the decision rule above, the similarity degree sequence for the 4 instances with the target instance is C3, C6, C1, C7, namely instance C3 is most similar with C0.

Table 2. Sorting Process of Property Parameter

\begin{tabular}{|c|c|c|c|c|c|c|c|c|c|c|c|c|c|c|}
\hline & \multicolumn{4}{|c|}{ DN } & \multicolumn{6}{|c|}{ Pressure } & \multicolumn{4}{|c|}{ Temperature } \\
\hline \multirow{4}{*}{$\stackrel{\theta}{\Xi}$} & 0 & 0.75 & 0.67 & 0.80 & \multirow{4}{*}{$\underset{\mathbb{N}}{\tilde{N}}$} & 0 & 0.41 & 0.43 & $0.29]$ & \multirow{4}{*}{$\underset{\omega}{\tilde{\omega}}$} & 0 & 0.20 & 0.33 & $0.64]$ \\
\hline & 0.25 & 0 & 0.4 & 0.57 & & 0.59 & 0 & 0.52 & 0.37 & & 0.80 & 0 & 0.67 & 0.86 \\
\hline & 0.33 & 0.60 & 0 & 0.67 & & 0.57 & 0.48 & 0 & 0.35 & & 0.67 & 0.33 & 0 & 0.78 \\
\hline & 0.20 & 0.43 & 0.33 & 0 & & 0.71 & 0.63 & 0.65 & 0 & & 0.36 & 0.13 & 0.22 & 0 \\
\hline \multirow{5}{*}{$\stackrel{E}{\Theta}$} & \multicolumn{4}{|c|}{$\lambda=0.5$} & \multicolumn{6}{|c|}{$\lambda=0.5$} & \multicolumn{4}{|c|}{$\lambda=0.6$} \\
\hline & & & $4 \quad 0$. & & \multirow{3}{*}{$\stackrel{\theta}{\hat{N}}$} & 0 & & 41 & & \multirow{3}{*}{ 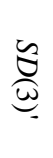 } & 0 & & $\begin{array}{ll}33 & 0.6\end{array}$ & \\
\hline & & $6 \quad 0$ & 0. & & & 0.5 & & 0. & & & 0.67 & $\begin{array}{ll}7 & 0\end{array}$ & 0.7 & \\
\hline & 0.43 & $3 \quad 0.3$ & 33 & & & 0.57 & 0. & 48 & & & 0.36 & $\begin{array}{ll}6 & 0.2\end{array}$ & 22 & \\
\hline & \multicolumn{4}{|c|}{$\lambda=0.6$} & \multicolumn{6}{|c|}{$\lambda=0.5$} & \multicolumn{4}{|c|}{$\lambda=0.6$} \\
\hline \multirow{2}{*}{$\underbrace{\mathscr{E}}_{=}$} & & & 0.57 & & \multirow{2}{*}{$\underbrace{\mathscr{N}}_{=}$} & \multirow{2}{*}{\multicolumn{4}{|c|}{$\left[\begin{array}{cc}0 & 0.43 \\
0.57 & 0\end{array}\right]$}} & \multirow{2}{*}{$\underset{=}{\stackrel{\sigma}{\omega}}$} & \multirow{2}{*}{\multicolumn{4}{|c|}{$\left[\begin{array}{cc}0 & 0.64 \\
0.36 & 0\end{array}\right]$}} \\
\hline & & 0.43 & 0 & & & & & & & & & & & \\
\hline$T_{1}$ & \multicolumn{4}{|c|}{$1,3,2,4$} & $T_{2}$ & \multicolumn{4}{|c|}{$4,2,3,1$} & $T_{3}$ & \multicolumn{4}{|c|}{$3,1,2,4$} \\
\hline
\end{tabular}

(3) Recycle Reuse Process by the Integrated System. Recycling reuse process can be formulated as follows: first of all, it should be done to import the simplified model of the valve body from UG system, and then making the structural analysis in ANSYS. In analysis, the finite element analysis reuse model *.mac file generated by APDL of the valve body could be imported from the reuse base, and then the necessary operation should be executed due to model modification, finally the information reuse of finite element analysis can be completed. As shown in Figure 4, if the analysis results don't meet the design requirements, it allows to input parameters under the UG environment to modify CAD model of the valve body, and then make re-analysis by ANSYS until getting the satisfied results. 


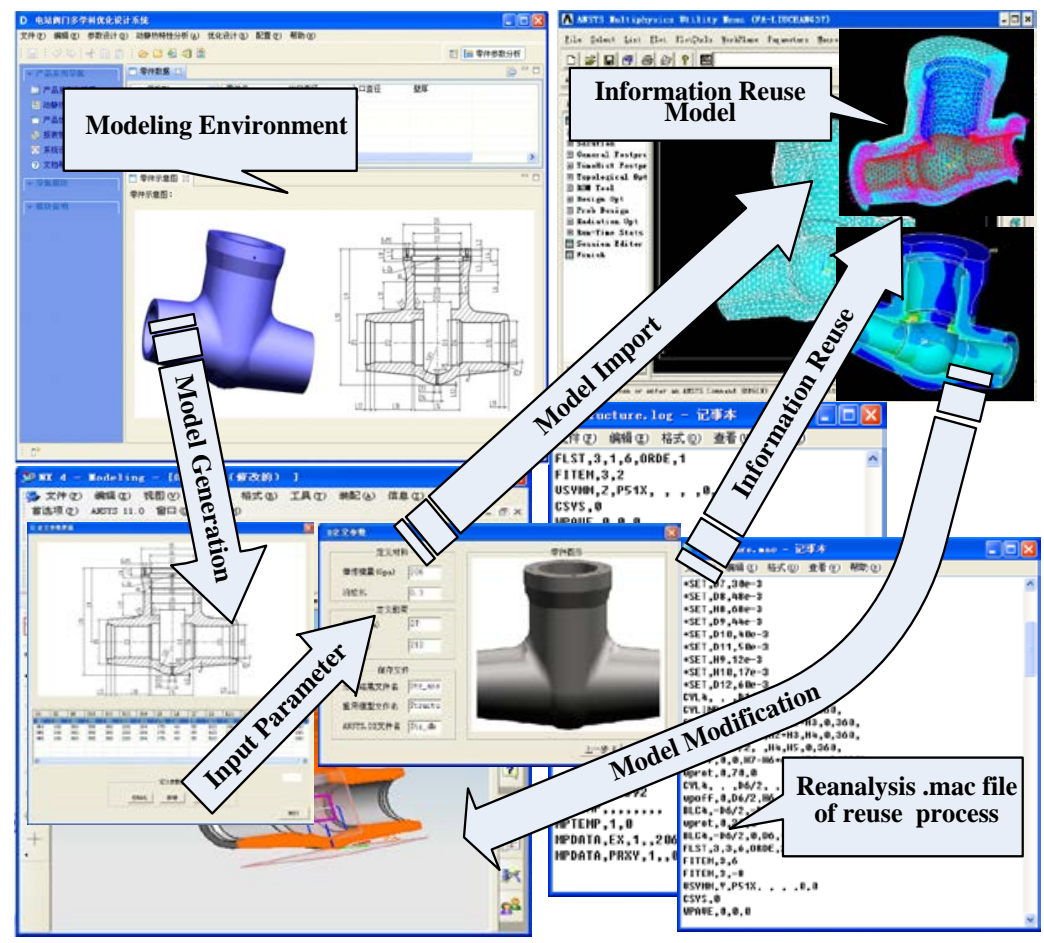

Figure 4. FEA Reuses Process in Heterogeneous CAD/CAE Environment

\section{Conclusion}

To achieve fast response to multi-characteristic FEA on unclear power valve, a kind of reuse technology of finite element analysis is proposed. Through extraction, integration, and reuse of existing analytical instance, reuse process of finite element analysis on valve body is realized, that effectively improves the simulation accuracy and efficiency.

(1) The finite element analysis integration model (FEAIM) is constructed by analyzing the information structure of finite element simulation analysis, and the information from of finite element simulation analysis is formally expressed.

(2) Heterogeneous information between CAD and FEA of valve models is expressed by using the universal data exchange technology, and reusable template description is given to realize the knowledge reuse of finite element analysis among similar models based on XML.

(3) Analytical cyclic reusable process is achieved by using APDL and relevant macro command and macro library. The integration platform of information reuse process for nuclear power valve is realized through secondary development of ANSYS and UG, which, moreover, could provide technical reference for the practicality and commercialization of the integrated system in valve products development.

\section{Acknowledgements}

This research was supported by the National Natural Science Foundation of China (Grant No. 51105120), the New Century Excellent Talents in University (Grant No.NCET-10-0146) and the Foundation for Distinguished Young Scholars of Heilongjiang Province, China (Grant No. JC201111). 


\section{References}

[1] S. M. Gao, W. Zhao and H. W. Lin, "Feature Suppression based CAD mesh model simplification", Computer-Aided Design, vol. 42, no. 12, (2010), pp. 1178-1188.

[2] Y. Zhang, H. Wang and H. Zhou, "Finite triangular surface mesh simplify-cation with geometrical feature recognition”, Journal of mechanical engi-neeering sciene, vol. 223, no. 11, (2009), pp. 2627-26.

[3] K. Y. Lee, C. G. Armstrong and M. A. Price, "A small feature suppression unsuppression system for preparing B-rep models for analysis”, The 2005 ACM symposium on solid and physical modeling, vol. 1315, (2005), pp. 113-124.

[4] H. Date, S. Kanai and T. Kisinami, "High-quality and property controlled finite element mesh generation from triangular meshes using the multiresolution technique”, Journal of Computing and Information Science in Engineering, vol. 5, no. 4, (2005), pp. 266-276.

[5] A. Thakur, A. G. Banerjee and S. K. Gupta, "A Survey of CAD Model Simplification Techniques for Physics-based Simulation Applications”, Computer-Aided Design, vol. 41, no. 2, (2009), pp. 65-80.

[6] P. Solin, J. Cerveny and I. Dolezel, “Arbitrary-level hanging nodes and automatic adaptivity in the hp-FEM”, Mathematics and computers in simulation, vol. 77, (2008), pp. 117-132.

[7] K. H. Chang and S. H. Joo, "Design parameterization and tool integration for CAD-based mechanism optimization”, Advances in Engineering Software, vol. 37, no. 12, (2006), pp. 779-796.

[8] J. H. Seungho, J. Lee and T. Lee, "Parametric study using an integrated design system to assess fatigue durability of control arms in automobile suspension module”, The 11th International Conference, vol. 5236, (2007), pp. 144-154.

[9] X. Zhu and Y. Xinhua, "The investigation of FEM software further-deverlopment model based on XML", Control \& Automation, (in Chinese), vol. 23, no. 21, (2007), pp. 216-219.

[10] Y. Xiaofeng, "Study on Stamping mould design knowledge reuse technology based on XML", Wuhan: Huazhong University of Science and Technology, (in Chinese), (2010), pp. 62-69.

[11] Y. Wei, W. Mingwen and W. Jianyi, "Positional Language Models with Semantic Information", Acta Scientiarum Naturalium Universitatis Pekinensis, (in Chinese), vol. 49, no. 2, (2013), pp. 203-212. 
International Journal of $\mathrm{u}$ - and e- Service, Science and Technology Vol.6, No.5 (2013) 